\title{
Oscillations of Andreev States in Clean Ferromagnetic Films
}

\author{
M. Zareyan, ${ }^{1,2}$ W. Belzig, ${ }^{1}$ and Yu. V. Nazarov ${ }^{1}$ \\ ${ }^{1}$ Department of Applied Physics and Delft Institute of Microelectronics and Submicrontechnology, \\ Delft University of Technology, Lorentzweg 1, 2628 CJ Delft, The Netherlands \\ ${ }^{2}$ Institute for Advanced Studies in Basic Sciences, 45195-159, Zanjan, Iran
}

(Received 5 October 2000)

\begin{abstract}
We investigate the influence of the exchange field on the Andreev bound states in a ferromagnetic $(F)$ film backed on one side by a superconductor $(S)$. Our model accounts for diffusive reflection at the outer surface and possible backscattering at the $F S$ interface. Phase shifting of the Andreev level by the exchange field results in an oscillatory behavior of the density of states of $F$ as a function of the layer thickness. We show that our results agree quantitatively with recent experiments.
\end{abstract}

DOI: $10.1103 /$ PhysRevLett.86.308

Probing the proximity effect by tunneling spectroscopy of induced superconducting correlations has a long history. Early experiments on the proximity density of states (DOS) [1] could be understood in the tunneling model of McMillan [2]. Recently the spatial dependence of the proximity density of states in normal metals has been measured [3] and successfully explained in terms of the quasiclassical theory [4]. The influence of spin splitting by a parallel magnetic field was measured in [5] and found to coincide with a Zeeman-split density of states. A new experimental and theoretical challenge is to extend these studies to the superconducting proximity effect in ferromagnets.

Previous experimental investigations have concentrated on thermodynamic properties of $F S$ multilayers. Here oscillations of the superconducting critical temperature $T_{\mathrm{c}}$ as a function of the thickness of the $F$ layers have been predicted [6] and found experimentally [7]. However, the interpretation of the experimental results depends on many fitting parameters. For example, little is known about sample parameters like the $F S$-interface quality [8]. In our opinion, it is also questionable if the theoretical approach using the diffusive quasiclassical formalism [9] is applicable for all $F$ layers of typical thicknesses $10-50 \AA$.

The most recent experiments have concentrated on other properties of $F S$ layers. Ryazanov et al. [10] studied the supercurrent through a thin ferromagnetic layer and found a nonmonotonic temperature dependence, which can be interpreted in terms of a $\pi$-phase shift due to the exchange splitting. Kontos et al. [11], on the other hand, have studied the DOS in a thin ferromagnetic layer in contact to a superconductor. An oscillatory behavior of the induced superconducting correlation was observed for layers of different thicknesses and attributed to influence of the exchange field. It is this experiment that motivated our present study.

Bearing this in mind, we study the superconducting proximity effect in a thin ferromagnetic layer. The $F$ film is characterized by an homogenous exchange splitting $h$. We model the film as a ballistic layer with rough boundaries. Band mismatch and disorder at the interface may
PACS numbers: 74.50.+r, 74.80.-g, 75.70.-i

lead to enhanced backscattering at the $F S$ boundary. We will derive a general formula for the subgap density of states depending only on the length distribution of classical trajectories in the $F$ layer. The resulting density of states shows as a signature of the exchange splitting an oscillatory behavior as a function of layer thickness. Comparison to the experimental data shows reasonable agreement keeping in mind the large uncertainty of some sample parameters.

The system we consider is sketched in Fig. 1. A thin ferromagnetic layer $(F)$ of thickness $d$ is connected to a superconducting bank $(S)$ on one side and bounded on the other side by an insulator. The $F$ layer is characterized by an exchange splitting, which we take into account as mean
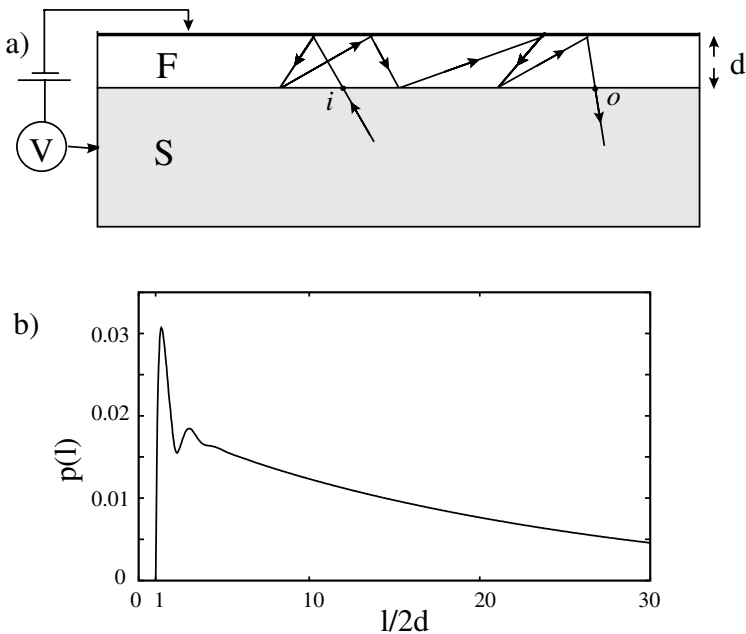

FIG. 1. (a) Schematic drawing of a ferromagnetic film in connection with a superconductor. A typical classical trajectory is also indicated. An electron coming from the bulk of the superconductor enters into the ferromagnet (at point $i$ ) and after several diffusive reflections from the insulator and the $S F$ interface returns to the superconductor (at point $o$ ). (b) The calculated distribution of the trajectory lengths in the $F$ layer for small $S F$ transparency (here $T=0.1$ ). The double peak structure close to the smallest length originates from the first two reflections from the insulator, whereas the distribution for long trajectories decays as $\exp (-l / \bar{l})$, with the average length $\bar{l} \approx 2 d / T$. 
field $h$ in the Hamiltonian. The thickness $d$ is larger than the Fermi wavelength $\lambda_{\mathrm{F}}$ and smaller than the elastic mean free path $\ell_{\text {imp }}$, which allows for a quasiclassical description [12] in the clean limit. Then, the real-time Eilenberger equation reads

$$
\begin{aligned}
-i \boldsymbol{v}_{\mathrm{F}} \boldsymbol{\nabla} \hat{g}_{\sigma}\left(E, \boldsymbol{v}_{\mathrm{F}}, \boldsymbol{r}\right)=[ & {[E+\sigma h(\boldsymbol{r})] \hat{\tau}_{3} } \\
& \left.-i \hat{\tau}_{2} \Delta(\boldsymbol{r}), \hat{g}_{\sigma}\left(E, \boldsymbol{v}_{\mathrm{F}}, \boldsymbol{r}\right)\right] .
\end{aligned}
$$

Here $\hat{\boldsymbol{\tau}}_{i}$ denote the Pauli matrices, $\Delta(\boldsymbol{r})$ is the (real) superconducting pair potential, and $\sigma(= \pm 1)$ labels the electron spin. The matrix Green's functions have to obey the normalization condition $\hat{g}_{\sigma}^{2}=1$. Inside the $F$ layer $h$ is constant and $\Delta=0$. We neglect the change of the pair potential in $S$, thus $\Delta(r)=$ const inside the superconductor. Strictly speaking, we would have to include an elastic collision term in (1), even in the limit $\ell_{\text {imp }} \gg d$. However, changes in the spectrum due to this term are limited to small energies $\lesssim v_{\mathrm{F}} / \ell_{\text {imp }} \ll \min \left(v_{\mathrm{F}} / d, h\right)$ [13], which are negligible in all cases that we study. Disorder in the superconductor can be neglected in the limit of small interface transmission [13] as in the experiment [11]. We have solved Eq. (1) along each classical trajectory in $F$ that comes from the superconductor and ends there. The DOS for $|E|<\Delta$ on a given trajectory of length $l$ is then given by

$$
\begin{aligned}
N(E, l) & =\frac{N_{0}}{4} \sum_{\sigma= \pm 1} \operatorname{Re}\left[\operatorname{Tr} \hat{\tau}_{3} \hat{g}_{\sigma}\left(E, \boldsymbol{v}_{\mathrm{F}}, \boldsymbol{r}\right)\right] \\
& =\frac{N_{0}}{2} \sum_{\sigma= \pm 1} \frac{\pi v_{\mathrm{F}}}{|E+\sigma h|} \sum_{n=-\infty}^{\infty} \delta\left(l-l_{n}\right),
\end{aligned}
$$

where

$$
l_{n}=\frac{v_{\mathrm{F}}}{E+\sigma h}[n \pi+\arccos (E / \Delta)] .
$$

Here $N_{0}$ is the density of states at the Fermi level in the normal state. Inside the $F$ layer $N(E, l)$ is constant along a given trajectory and depends only on the length of the trajectory $l$. Equation (2) means that the DOS below $\Delta$ is a sum of $\delta$ peaks resulting from Andreev bound states. The energies follow from the quasiclassical quantization condition $l=l_{n}$. The total DOS can then be found by averaging (2) over all classical trajectories. Denoting the trajectory length distribution $p(l)$ we find for the density of states

$$
N(E)=\frac{N_{0}}{2} \sum_{\sigma= \pm 1} \frac{\pi v_{\mathrm{F}}}{|E+\sigma h|} \sum_{n=-\infty}^{\infty} p\left(l_{n}\right) .
$$

This formula presents the general result for subgap density of states of a quasiballistic ferromagnet connected to a superconductor. It is completely specified by the length distribution of classical trajectories. The distribution depends only on the geometrical properties of the attached ferromagnet and the connecting interface.

Now we have to specify the trajectory length distribution for our particular case. We model the thin layer by a weakly disordered thin film with a rough surface and a rough $S F$ interface of average transparency $T$. A typical trajectory is depicted in Fig. 1. An electron coming from the bulk of $S$ enters into the $F$ layer and after several reflections from the insulator and the $F S$ interface returns to the $S$ bank (see Fig. 1), where it is Andreev reflected as a hole which traverses the trajectory in the opposite direction. Thus, the elementary building block of a typical trajectory is the segment between two successive reflections from the superconductor. The number of blocks, which form the total trajectory, depends on the transparency of the interface, i.e., it is roughly $\sim 1 / T$. First, let us consider the length distribution of one elementary block. Because of the roughness of the insulator and the $F S$ interface the quasiparticles undergo diffusive reflection from these boundaries. Incoming and outgoing directions are completely uncorrelated. Accounting for the weak bulk disorder we include a factor $\exp \left(-l / \ell_{\text {imp }}\right)$ in the length distribution, which mainly serves to give a finite value of the average length. In a purely ballistic layer the average length would be logarithmically divergent. The length distribution of one elementary block is then given by

$$
\begin{aligned}
p_{0}(l)= & \frac{2 d}{C l^{2}}\left[\frac{l-2 d}{l-d}+\frac{2 d}{l} \ln \frac{l-d}{d}\right] \\
& \times e^{-l / \ell_{\text {imp }}} \theta(l / d-2),
\end{aligned}
$$

where $C=E_{2}^{2}\left(d / \ell_{\text {imp }}\right)\left[E_{2}(z)=\int_{1}^{\infty} d x \exp (-z x) / x^{2}\right.$ is the exponential integral of second order]. Second, we connect the elementary building blocks, if the $S F$ interface has an average transparency $T$. In determining the length distribution we assume that a particle either goes through the interface or is fully reflected. Only the number of these reflections depends on $T$. We do not take into account quantum mechanical interference for a single reflection at the $F S$ interface. Taking this into account will yield essentially the same results as our approach. By an expansion in the reflectivity $R=1-T$ it is easy to see that the full distribution $p(l)$ obeys the integral equation

$$
p(l)=T p_{0}(l)+R \int d l^{\prime} p_{0}\left(l^{\prime}\right) p\left(l-l^{\prime}\right) .
$$

This is readily solved by a Fourier transformation. We obtain

$$
p(l)=\int_{-\infty}^{\infty} \frac{d k}{2 \pi} e^{i k l} \frac{T P_{0}(k)}{1-R P_{0}(k)},
$$

where $P_{0}(k)=E_{2}^{2}\left(i k d+d / \ell_{\text {imp }}\right) / C$ is the Fourier transform of $p_{0}(l)$. The distribution $p(l)$ is plotted for $T=0.1$ and $d / \ell_{\text {imp }}=0.1$ in Fig. 1 . It has a double peak structure for short trajectories $l \geqslant 2 d$ resulting from trajectories reflected once or twice from the insulator. At large $l$ the distribution decays exponentially as $\exp (-l / \bar{l})$, where $\bar{l} \simeq 2 d \ln \left(\ell_{\mathrm{imp}} / d\right) / T$ is the mean trajectory length. For $T \sim 1, p(l)$ has only one peak close to $2 d$. We therefore have two characteristic lengths of the distribution, the smallest possible trajectory length $2 d$ and the average 
length $\bar{l}$. Which of these length scales will determine the total DOS will depend on the other parameters, in particular, on $h$.

Combining Eqs. (2), (4), and (7) we obtain for the total DOS

$$
N(E)=\frac{1}{2} \sum_{\sigma= \pm} \sum_{n=-\infty}^{\infty} \frac{T P_{0}\left(k_{n}\right)}{1-R P_{0}\left(k_{n}\right)} e^{2 n i \arccos E / \Delta} N_{0},
$$

where $k_{n}=2 n(E+\sigma h) / v_{\mathrm{F}}$. Thus, the density of states is fully expressed in terms of known functions. In the following we will discuss the parameter range of rather strong exchange fields in the limit of thin layers $d \ll v_{\mathrm{F}} / \Delta$. The results presented below are in the most realistic case of small $S F$-interface transparency $T \ll 1$. In this limit the distribution of long lengths is well approximated as $\exp (-l / \bar{l})$. Most probably the $F$ film has a nonuniform thickness due to the large scale roughness of the boundaries. For a smoothly varying thickness we can take this into account by averaging (8) over a Gaussian distribution of the thicknesses around a mean value $d$. This will also lead to a smoothening of the sharp features in the DOS resulting from the lower cutoff in $p(l)$. The qualitative behavior will however not change. In practice, we have chosen a width of the distribution to be of the order of $10 \%$ that corresponds to the condition of the experiment [11].

For weak exchange fields when $h \bar{l} / v_{\mathrm{F}} \lesssim 1$ the DOS at low energies $E \ll \Delta$ is mainly contributed from long trajectories with $l \gtrsim \bar{l}$. The precise form of the DOS in this regime will therefore depend on $\ell_{\text {imp }}$, whereas qualitative features as discussed below are independent of $\ell_{\text {imp. }}$. The DOS versus $h d / T v_{\mathrm{F}}$ is plotted for dif-

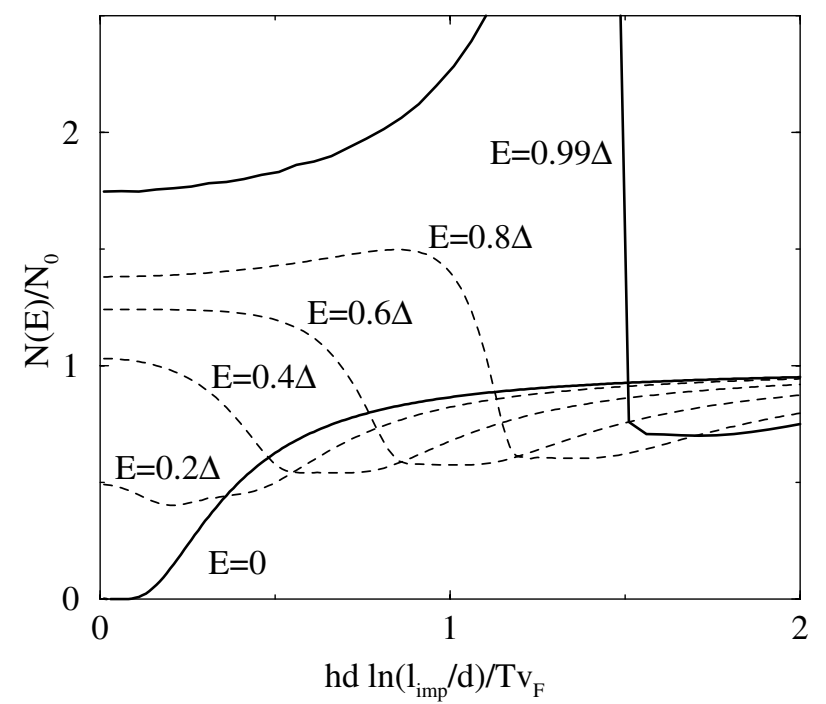

FIG. 2. Density of states for weak exchange fields and small $S F$-interface transparency. For $E=0$ the curve is independent of $T$ as long as $T \ll 1$. At finite energies the precise form depends on the transparency (here $T=0.03$ and $\ell_{\text {imp }}=100 d$ ). It has a broad peak at zero energy and two dips $E= \pm h$ resulting from the shifts of the Andreev bound states. ferent energies in Fig. 2. For $h=0$ (a normal film), $N(E)$ vanishes at zero energy and increases for finite energies ending with a peak at $\Delta$. The effect of the exchange field in the regime $h<\Delta$ is to split the DOS for the two spin bands. Thus the total DOS is the average of two by $\pm h$ shifted spectra resulting in a peak at zero energy and two dips at $E= \pm h$. Increasing $h$ further leads to a suppression of superconducting features of the DOS. The zero energy DOS increases roughly as $\left(\pi v_{\mathrm{F}} / h \bar{l}\right) \exp \left(-\pi v_{\mathrm{F}} / 2 h \bar{l}\right) /\left[1-\exp \left(-\pi v_{\mathrm{F}} / h \bar{l}\right)\right]$, which follows from the approximation mentioned above. At finite energies $N(E)$ passes through minima corresponding to $E= \pm h$ before approaching the normal metal DOS.

For larger exchange fields when $h d v_{\mathrm{F}} \gtrsim 1$ mainly the short trajectories of $l \sim 2 d$ contribute to the energy dependence of $N(E)$. The DOS is close to that of the normal state. The interesting parts are the small deviations of amplitude $T$ which oscillate as a function of $h d$. In Fig. 3 we have plotted $\left[N(E) / N_{0}-1\right] / T$ versus $h d / v_{\mathrm{F}}$ for different energies. At small $h d / v_{\mathrm{F}} \ll 1$ and $E \ll \Delta$ the DOS as a function of $h d / v_{\mathrm{F}}$ still reminds one of the double peak structure of the length distribution. For $h d / v_{\mathrm{F}} \gtrsim 1$, however, it develops coherent oscillations as a function of $h d / v_{\mathrm{F}}$ with a period of $\pi / 2$. The magnitude and the sign of the oscillation depend on the energy. Maximal amplitudes of opposite sign always correspond to $E=0$ or $|E|=\Delta$. It is worth noting that neither the amplitude nor the period of the oscillations depends on the mean free path $\ell_{\text {imp }}$.

Let us now discuss the relation of our results to the recent experiment performed in the Orsay group [11]. They observed DOS oscillation in thin films of the ferromagnetic

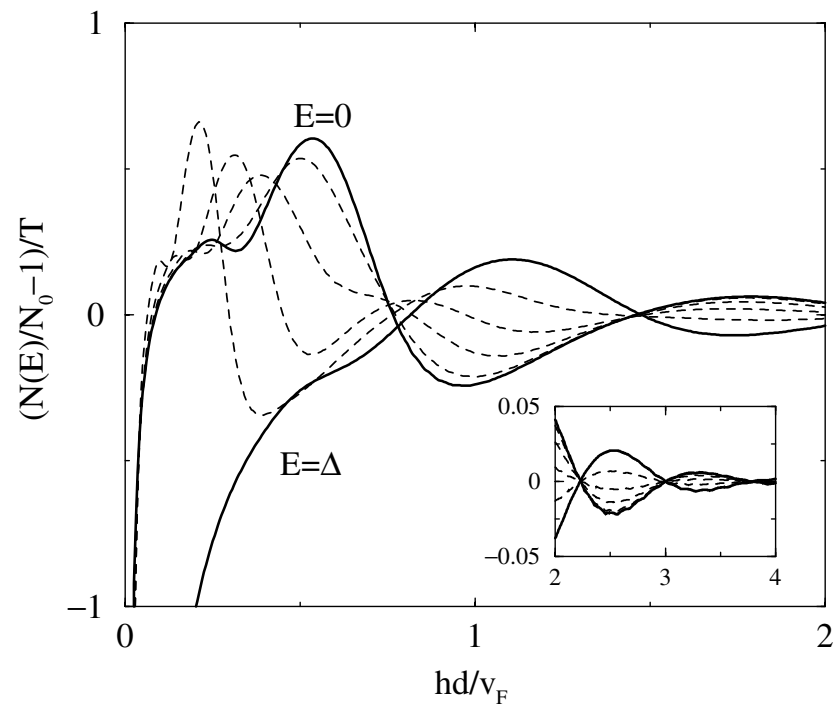

FIG. 3. Oscillation of reduced DOS $\left[N(E) / N_{0}-1\right] / T$ with the exchange field. For $h d / v_{\mathrm{F}} \gtrsim 1$, it develops coherent oscillations as a function of $d h / v_{\mathrm{F}}$ with a period of $\pi / 2$ and an energy dependent magnitude and sign. Maximal amplitudes of opposite sign always correspond to $E=0$ or $|E|=\Delta$. The inset shows how the oscillation continues for $h d / v_{\mathrm{F}}>2$. 


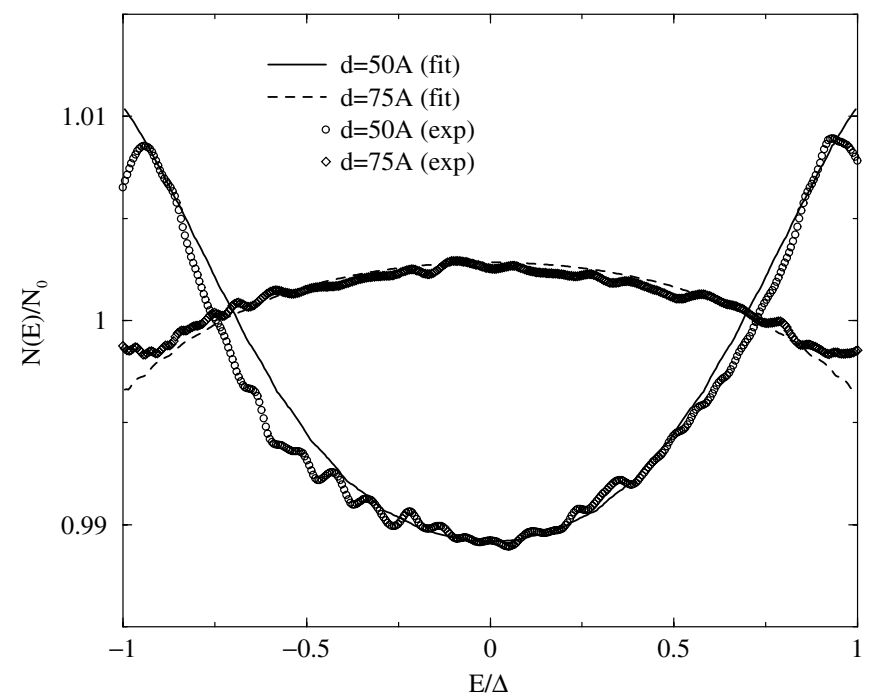

FIG. 4. Energy dependence of DOS for two different thicknesses of the ferromagnetic film, $d_{1}=50 \AA$ (solid line) and $d_{2}=75 \AA$ (dashed line), with the corresponding experimental curves of Ref. [11]. The DOS oscillation appears as an inversion of the energy dependence of the DOS when $d$ changes by $\pi v_{\mathrm{F}} / 4 h$.

alloy $\mathrm{Pd}_{1-x} \mathrm{Ni}_{x}$ with $x$ of the order of $10 \%$ in contact with a $\mathrm{Nb}$ electrode. By tunneling spectroscopy they measured the differential conductance versus bias of the $F$ film at low temperatures $\sim 300 \mathrm{mK}$. Normalized to the normal state conductance this yields the density of states in the ferromagnetic layer. For two different thicknesses of the $F$ film, $d_{1}=50 \AA$ and $d_{2}=75 \AA$ the reduced density of states differs in sign and magnitude, but has the same functional energy dependence. The exchange field was estimated by several methods to be in the range $h=5-15 \mathrm{meV}$. These experimental determinations were, however, only sensitive to the average magnetization. In thin layers, it is reasonable to assume that the magnetic structure has multiple domains, inhomogeneous thickness (as already discussed before), and nonuniform $\mathrm{Ni}$ concentration. All these effects tend to suppress the measured values of the exchange field below the value, which results from the local Ni-doping level. We will therefore regard the experimental values of $h$ as lower bounds to the value used in our fits. In Fig. 4 we compare the data of Ref. [11] with our calculation. We have taken $v_{\mathrm{F}}=2 \times 10^{7} \mathrm{~cm} / \mathrm{s}$ and $h=29 \mathrm{meV}$, and $T=0.055$. Our value of the exchange field has been deduced numerically from the ratio of the magnitudes of $N(0) / N_{0}-1$ for the two different thicknesses. The agreement between the experimental data and our calculation is satisfactory. Both the functional dependence and the sign change are correctly reproduced by our calculations.

In conclusion we have investigated theoretically the superconducting proximity effect in thin ferromagnetic layers in a quasiballistic model. We have found that the effect of the ferromagnet exchange field $h$ is to suppress the superconducting features in the density of states for $d h / v_{\mathrm{F}} \gtrsim T$. At exchange fields larger than $v_{\mathrm{F}} / d$ the density of states oscillates around the normal state value as a function of $d h / v_{\mathrm{F}}$ with a period of $\pi / 2$ and an amplitude of the order of the interface transparency $T$. We have shown that the oscillation can lead to an inverted energy dependence of the DOS. This effect has been observed in the experiment of Ref. [11]. We have achieved quantitative agreement between our theory and the experimental data.

We thank T. Kontos and M. Aprili for sharing their data with us prior to publication, which was the initiation of this work. W. B. was financially supported by the "Stichting voor Fundamenteel Onderzoek der Materie" (FOM) and the "Alexander von Humboldt-Stiftung." M. Z. gratefully acknowledges financial support from the Iranian Ministry of Science, Research and Technology and the TU Delft.

Note added in proof.-After submission an article [14] appeared, in which results similar to ours were found with a diffusive quasiclassical approach. As mentioned earlier, we do not believe that the diffusive approximation is justified, since typically $l \gtrsim v_{\mathrm{F}} / h$ [11]. In addition the measured mean free paths of the ferromagnetic films are roughly given by the respective film thickness [15] as expected for clean films with rough surfaces.

[1] W. L. McMillan and J.R. Rowell, in Superconductivity, edited by R. D. Parks (Marcel Dekker, New York, 1969).

[2] W. L. McMillan, Phys. Rev. 175, 537 (1968).

[3] S. Guéron et al., Phys. Rev. Lett. 77, 3025 (1996).

[4] W. Belzig et al., Phys. Rev. B 54, 9443 (1996).

[5] W. J. Gallagher et al., Phys. Rev. B 21, 962 (1980); R. Meservey and P.M. Tedrow, Phys. Rep. 238, 173 (1994).

[6] Z. Radović et al., Phys. Rev. B 44, 759 (1991).

[7] J.S. Jiang et al., Phys. Rev. Lett. 74, 314 (1995); Th. Mühge et al., ibid. 77, 1857 (1996).

[8] J. Aarts et al., Phys. Rev. B 56, 2779 (1997); L. Lazar et al., ibid. 61, 3711 (2000); M. Schöck et al., Eur. Phys. J. B 14, 1 (2000).

[9] K. D. Usadel, Phys. Rev. Lett. 25, 507 (1970).

[10] A. V. Veretennikov et al., Physica (Amsterdam) 284B-288B, 495 (2000); V. V. Ryazanov et al., cond-mat/ 0008364 (unpublished).

[11] T. Kontos et al., preceding Letter, Phys. Rev. Lett. 86, 304 (2001).

[12] G. Eilenberger, Z. Phys. 214, 195 (1968); A. I. Larkin and Yu. N. Ovchinnikov, Sov. Phys. JETP 26, 1200 (1968).

[13] S. Pilgram et al., Phys. Rev. B 62, 12462 (2000).

[14] A. Buzdin, Phys. Rev. B 62, 11377 (2000).

[15] M. Aprili (private communication). 